\title{
Las funciones esenciales de la salud pública: un tema emergente en las reformas del sector de la salud
}

\author{
Fernando Muñoz, ${ }^{1}$ Daniel López-Acuña, ${ }^{2}$ Paul Halverson ${ }^{3}$ \\ Carlyle Guerra de Macedo, ${ }^{4}$ Wade Hanna, ${ }^{5}$ Mónica Larrieu, ${ }^{6}$ \\ Soledad Ubilla, ${ }^{7}$ y José Luis Zeballos ${ }^{8}$
}

RESUMEN En las Américas, las reformas del sector de la salud se enfrentan al desafío de fortalecer la función rectora de las autoridades sanitarias y una parte importante de este papel consiste en dar cumplimiento a las funciones esenciales de la salud pública (FESP) que competen al Estado en sus niveles central, intermedio y local. Para ello es crucial mejorar la práctica de la salud pública y los instrumentos para valorar su estado actual y las áreas en las que debe ser fortalecida. En virtud de lo anterior, la Organización Panamericana de la Salud (OPS) ha puesto en marcha la iniciativa "La salud pública en las Américas", dirigida a la definición y medición de las FESP como base para mejorar la práctica de la salud pública y fortalecer el liderazgo de las autoridades sanitarias en todos los niveles del Estado. El presente artículo resume aspectos conceptuales y metodológicos relacionados con la definición y medición de las FESP y analiza las implicaciones de la medición del desempeño de las mismas en el mejoramiento de las prácticas de la salud pública en las Américas.

En las Américas, los procesos de reforma del sector de la salud se han

1 Centro Latinoamericano de Investigación en Sistemas de Salud (CLAISS). Toda la correspondencia debe ser enviada a Fernando Muñoz a la siguiente dirección postal: CLAISS, José Miguel de la Barra 412, Piso 3, Santiago, Chile. Correo electrónico: claiss@ctcreuna.cl

2 División de Desarrollo de Sistemas y Servicios de Salud, Organización Panamericana de la Salud/ Organización Mundial de la Salud (OPS/OMS), Washington, D.C., Estados Unidos de América.

3 CDC, Atlanta, Estados Unidos de América.

4 Director Emérito, OPS/OMS, Washington, D.C., Estados Unidos de América.

5 Centros para el Control y Prevención de Enfermedades (CDC), Atlanta, Estados Unidos de América.

6 OPS/OMS, Washington, D.C., Estados Unidos de América.

7 CLAISS, Santiago, Chile.

8 Programa de Organización y Gestión de Sistemas y Servicios de Salud, OPS/OMS, Washington, D.C., Estados Unidos de América. concentrado principalmente en los cambios estructurales, financieros $\mathrm{y}$ organizacionales de los sistemas de salud y en los ajustes a la prestación de servicios de atención a las personas. La salud pública ha sido descuidada como una responsabilidad social e institucional, justamente cuando más atención requiere y cuando más se necesita apoyo de los gobiernos para modernizar la infraestructura necesaria para su ejercicio (1).

La reinserción de la salud pública en la agenda de transformación del sector pasa por la definición clara de su papel (2) y por la operacionalización de los conceptos que le dan fundamento, entre ellos el de funciones esenciales de la salud pública (FESP) (3). Para la recuperación de la salud pública y para su inserción en los procesos de transforma- ción del sistema, también es importante que estas categorías operacionales, como las FESP, puedan ser caracterizadas y medidas, a fin de determinar su grado de cumplimiento, tanto por parte del Estado como de la sociedad civil (4).

Las reformas del sector se enfrentan al desafío de fortalecer la función de rectoría de las autoridades sanitarias y una parte importante de ese papel rector consiste en dar cumplimiento a las FESP que competen al Estado en sus niveles central, intermedio y local. Para ello es crucial mejorar la práctica de la salud pública y los instrumentos para valorar su estado actual y las áreas en las que debe ser fortalecida (5).

En virtud de lo anterior, la Organización Panamericana de la Salud (OPS) ha dado los pasos iniciales para poner en marcha una iniciativa continental 
denominada "La salud pública en las Américas", dirigida a la definición y medición de las FESP como base para mejorar la práctica de la salud pública y fortalecer el liderazgo de las autoridades sanitarias en todos los niveles del Estado. Esta iniciativa, que está siendo coordinada por la División de Sistemas y Servicios de Salud, involucra a todas las unidades técnicas de la sede así como a las representaciones de la OPS en los países, ha contado con la participación del Director Emérito de la OPS, Dr. Carlyle Guerra de Macedo, como asesor del proyecto y ha desarrollado los instrumentos de medición del desempeño de las FESP en conjunto con los Centros para el Control y Prevención de Enfermedades (Centers for Disease Control: CDC) de los Estados Unidos de América (EUA) y el Centro Latinoamericano de Investigación en Sistemas de Salud (CLAISS). El proyecto ha contemplado diversas instancias de interacción con expertos procedentes del medio académico, sociedades científicas, servicios de salud y organismos internacionales, congregados en una red de retroalimentación continua a su desarrollo, y está representando una valiosa oportunidad para revisar el estado actual de la práctica de la salud pública en la Región de las Américas.

El alcance de la iniciativa "La salud pública en las Américas" puede resumirse de la siguiente manera:

- promover un concepto común de la salud pública y de sus funciones esenciales en las Américas;

- crear un marco para la medición del desempeño de las FESP aplicable a todos los países americanos;

- apoyar la evaluación de la práctica de la salud pública en cada país, basándose en la medición del desempeño de las FESP;

- proponer un plan continental de acción para fortalecer la infraestructura y mejorar la práctica de la salud pública, basado en los hallazgos de la medición del desempeño de las FESP;

- publicar en el primer semestre de 2001 El estado de la salud pública en las Américas, un libro en el que se reunirán los diferentes resultados del proyecto y se ofrecerá un panorama del grado de cumplimiento de las FESP en las Américas.

A continuación se presentan algunos de los aspectos conceptuales y metodológicos que han sido formulados como parte de la iniciativa "La salud pública en las Américas", con especial énfasis en la definición y medición de las FESP que deben ser desarrolladas y que constituyen el quehacer y la responsabilidad de las autoridades sanitarias de los países del continente. Asimismo se analizan las implicaciones que tiene la medición del desempeño de las FESP para mejorar la práctica de la salud pública en nuestro continente (6).

\section{EL CONCEPTO DE FESP Y SU RELACIÓN CON EL FORTALECIMIENTO DE LA FUNCIÓN RECTORA DE LAS AUTORIDADES SANITARIAS}

El concepto de salud pública en el que se basa la definición de las FESP es el de la acción colectiva, tanto del Estado como de la sociedad civil, encaminada a proteger y mejorar la salud de las personas. Supone una noción que va más allá de las intervenciones poblacionales o comunitarias e incluye la responsabilidad de asegurar el acceso a la atención de salud y su calidad. No está referido a la salud pública como disciplina académica, sino como una práctica social de naturaleza interdisciplinaria. La salud pública no es sinónimo de responsabilidad del Estado en materia de salud, ya que su quehacer va más allá de las tareas propias del Estado y no abarca todo lo que el Estado puede hacer en materia de salud.

\section{Definición}

Las FESP se han definido como las condiciones que permiten un mejor desempeño de la práctica de la salud pública.

Una de las decisiones más importantes de la iniciativa "La salud pública en las Américas" está relacionada con la necesidad de adecuar la defini- ción de los indicadores y estándares de las FESP para permitir que la práctica de la salud pública se fortalezca a través del refuerzo de las capacidades institucionales necesarias. Este enfoque parece mejor que un planteamiento metodológico que incluya tanto funciones como campos de la actividad de la salud pública. Si las funciones son bien definidas para incluir todas las capacidades requeridas para una buena práctica de la salud pública, estará asegurado el buen funcionamiento en cada uno de los campos de acción o áreas de trabajo de la salud pública (cuadro 1).

Es importante referirse aquí a la frecuente confusión entre el papel del Estado en salud, ejercido normalmente por el Ministerio de Salud o su equivalente como autoridad sanitaria, y la responsabilidad del mismo como garante del buen desarrollo de las FESP. Si bien el Estado tiene un papel indelegable en la entrega directa o en la garantía del cumplimiento de las FESP, estas solo representan una fracción de las responsabilidades estatales en el ámbito de la salud. Es una fracción muy relevante, sin duda, cuyo adecuado cumplimiento resulta fundamental no solo para elevar los niveles de salud y la calidad de vida de la población, sino que es parte de la responsabilidad rectora que el Estado tiene en materia de salud, caracterizada asimismo por tareas de conducción, regulación, modulación del financiamiento, vigilancia del aseguramiento y armonización de la provisión. Para enfatizarlo con un ejemplo, una agencia de salud pública que no cuenta con un sistema integral y confiable de vigilancia de eventos en salud difícilmente puede aspirar a ser creíble cuando opina o actúa en materia de asignación financiera a los distintos componentes o sectores del sistema de salud.

También es relevante referirse aquí a la dificultad que entraña el establecimiento de una separación nítida entre las responsabilidades propias de la salud pública en la conducción de servicios dirigidos a la prevención de enfermedades y a la promoción de la salud en grupos poblacionales definidos, y aquellas que tienen que ver con la or- 
CUADRO 1. Funciones esenciales y campos de acción de la salud pública

\begin{tabular}{|c|c|c|c|c|c|}
\hline \multirow[b]{2}{*}{$\begin{array}{c}\text { FESP } \\
\text { (ejemplos) }\end{array}$} & \multicolumn{5}{|c|}{ Ámbitos de aplicación de las FESPa } \\
\hline & $\begin{array}{c}\text { Salud } \\
\text { ambiental }\end{array}$ & $\begin{array}{c}\text { Salud } \\
\text { ocupacional }\end{array}$ & $\begin{array}{l}\text { Salud } \\
\text { maternoinfantil }\end{array}$ & $\begin{array}{l}\text { Enfermedades } \\
\text { crónicas }\end{array}$ & Otras \\
\hline $\begin{array}{l}\text { Monitoreo de la } \\
\text { situación de salud }\end{array}$ & $\begin{array}{l}\text { Monitoreo de los riesgos } \\
\text { ambientales }\end{array}$ & $\begin{array}{l}\text { Monitoreo de los riesgos } \\
\text { en los lugares de } \\
\text { trabajo }\end{array}$ & $\begin{array}{l}\text { Monitoreo de los riesgos } \\
\text { en problemas de salud } \\
\text { maternoinfantil }\end{array}$ & $\begin{array}{l}\text { Monitoreo de los riesgos } \\
\text { de enfermedades } \\
\text { crónicas }\end{array}$ & \\
\hline $\begin{array}{l}\text { Fiscalización y } \\
\text { regulación }\end{array}$ & $\begin{array}{l}\text { Fiscalización del } \\
\text { cumplimiento de } \\
\text { normativas } \\
\text { ambientales }\end{array}$ & $\begin{array}{l}\text { Fiscalización de las } \\
\text { leyes referentes a la } \\
\text { protección del } \\
\text { trabajador }\end{array}$ & $\begin{array}{l}\text { Vigilancia del cumpli- } \\
\text { miento de las leyes } \\
\text { que protegen a la } \\
\text { madre y al hijo }\end{array}$ & $\begin{array}{l}\text { Fiscalización y regula- } \\
\text { ción para estimular } \\
\text { conductas saludables } \\
\text { que prevengan mayor } \\
\text { daño a la salud }\end{array}$ & \\
\hline
\end{tabular}

a FESP: funciones esenciales de la salud pública.

ganización de servicios dirigidos a la atención curativa individual. Los énfasis en esta materia son sin duda diferentes. Es patrimonio esencial de la salud pública el dedicarse a la primera de estas funciones. En lo que respecta a la segunda, sus responsabilidades esenciales apuntan más a la preocupación por el acceso equitativo a los servicios, la garantía de su calidad y la incorporación de la perspectiva de la salud pública a las políticas nacionales de salud. Esto no impide que los salubristas se capaciten para gestionar servicios de atención a los pacientes. Por el contrario, resulta deseable que lo hagan, precisamente para incorporar los conceptos de la salud pública al quehacer de tales organizaciones. Esta última actividad utiliza, sin embargo, disciplinas que trascienden a lo que damos en llamar "salud pública".

La habitual concepción de la salud pública como sinónimo del quehacer de salud contribuye a una dilución de las responsabilidades en diferentes ámbitos de esta última actividad y puede conducir a un uso ineficiente de los recursos de salud. La medición de las FESP y la evaluación de su desempeño por parte de las autoridades sanitarias debería contribuir a evitar este riesgo.

\section{Antecedentes}

En los últimos años se han llevado a cabo importantes esfuerzos para bus- car una mejor definición y medición de las FESP. Entre ellos cabe destacar el estudio Delphi conducido por la Organización Mundial de la Salud (OMS) y el Programa Nacional de Desempeño y Estándares de la Salud Pública (National Public Health Performance Standards Program: NPHPSP) de los EUA. A continuación se reseñan brevemente estas iniciativas.

\section{El estudio Delphi de la OMS sobre FESP}

En enero de 1997, el Consejo Ejecutivo de la OMS recomendó impulsar el desarrollo conceptual de las FESP como forma de contar con una herramienta para implementar la renovación de la política de "salud para todos en el año 2000". Para ello se decidió realizar un estudio internacional con la técnica Delphi, dirigido a redefinir el concepto de FESP y a obtener un consenso internacional sobre las características centrales de estas funciones (7). En ese estudio se consultaron en tres rondas consecutivas 145 expertos en salud pública de diferentes nacionalidades. El resultado fueron nueve FESP, a saber:

1. Prevención, vigilancia y control de enfermedades transmisibles y no transmisibles.

2. Monitoreo de la situación de salud.

3. Promoción de la salud.
4. Salud ocupacional.

5. Protección del ambiente.

6. Legislación y regulación en salud pública.

7. Gestión en salud pública.

8. Servicios específicos de salud pública.

9. Atención de salud para grupos vulnerables y poblaciones de alto riesgo.

Los autores recomendaron encarecidamente la realización de nuevos estudios nacionales e internacionales. También se resaltaron algunos temas controvertidos, como la relación entre los servicios de atención de salud y las FESP, área donde no hubo consenso en torno al grado en que la atención de salud individual de pacientes puede ser considerada una función básica y esencial de la salud pública.

\section{El Programa Nacional de Desempeño y Estándares de la Salud Pública (NPHPSP) de los EUA}

El informe de 1998 sobre salud pública de los Institutos Nacionales de Salud (National Institutes of Health: $\mathrm{NIH})$ de los EUA, producido después de más de 60 años de diferentes intentos de definir y medir las funciones de la salud pública y de evaluar el funcionamiento de las agencias de salud pública, definió tres grupos de funciones (evaluación, desarrollo de políticas y aseguramiento) para las organizacio- 
nes de salud pública, y entregó un marco racional para muchos de los esfuerzos dirigidos a evaluar los servicios de salud pública realizados en la última década en ese país (8).

En 1994, un grupo de trabajo sobre las principales funciones de la salud pública, codirigido por el Director de los CDC y el Secretario Adjunto para el Control de Enfermedades Crónicas y Promoción de la Salud y compuesto por representantes de las agencias de servicios de salud pública y las principales organizaciones de salud pública del país, señaló la confusión causada por las múltiples versiones de las "principales funciones" y encargó a un subgrupo encabezado por la Oficina del Programa de Prácticas en Salud Pública (Public Health Practice Program Office) y el Centro Nacional de Prevención de Enfermedades Crónicas y Promoción de la Salud (National Center for Chronic Disease Prevention and Health Promotion) de los CDC, el desarrollo de un consenso sobre los "servicios esenciales de salud pública"9 ". Este grupo elaboró una declaración de consenso que pretende: 1) explicar qué es la salud pública; 2) clarificar el papel esencial de la salud pública en todo el sistema de salud, y 3 ) dar transparencia a la vinculación del funcionamiento de la salud pública con los resultados de salud (9).

En 1994, el Comité Directivo de Funciones de la Salud Pública (Public Health Functions Steering Committee) adoptó el documento "Salud pública en los Estados Unidos de América" (10), en el que la visión "personas sanas en comunidades sanas" y la misión de promover la salud física y mental y prevenir las enfermedades, daños y discapacidades se sostienen en los siguientes objetivos principales de la salud pública:

- Prevención de epidemias y de la propagación de enfermedades.

- Protección contra los daños ambientales.

- Prevención de daños a la salud.

\footnotetext{
9 A efectos de esta publicación, el término "servicio esencial de salud pública" acuñado por el proyecto estadounidense, se homologa al de "función esencial de salud pública".
}

- Promoción y fomento de conductas saludables.

- Respuesta a los desastres y asistencia a comunidades damnificadas.

- Garantía de calidad y accesibilidad a los servicios de salud.

En el mismo documento se definieron 10 servicios esenciales de salud pública:

1. Monitoreo del estado de salud para identificar los problemas de salud de la comunidad.

2. Diagnóstico e investigación de los problemas de salud y de los riesgos en la comunidad.

3. Información, educación y empoderamiento de las personas en temas de salud.

4. Movilización de asociaciones comunitarias para identificar y resolver problemas de salud.

5. Desarrollo de políticas y planes que apoyen la salud individual y colectiva.

6. Cumplimiento de leyes y regulaciones que protejan la salud y den seguridad.

7. Vinculación de las personas con servicios de atención de salud y garantía de provisión de servicios de salud donde no estén disponibles.

8. Garantía de recursos humanos competentes para la salud pública y la atención de salud.

9. Evaluación de la eficacia, accesibilidad y calidad de los servicios de salud individual y colectiva.

10. Investigación sobre nuevos enfoques y soluciones innovadoras para los problemas de salud.

Como se puede ver, estos servicios pueden agruparse en cada una de las funciones principales definidas previamente. Los servicios 1 y 2 se incluyen en la función de evaluación; los servicios 3, 4 y 5 en la de desarrollo de políticas, y los servicios 6, 7, 8 y 9 en la de aseguramiento. El servicio 10 (investigación) se considera relacionado con todas las funciones principales como base para la gestión del sistema de salud pública (10).

Actualmente, el NPHPSP está liderando un esfuerzo conjunto para el de- sarrollo de estándares para la práctica de la salud pública. Las herramientas de medición para la evaluación de la práctica de la salud pública, tanto en el nivel local como estatal, han sido diseñadas en conjunto con otras organizaciones de salud pública. Estos instrumentos son cuestionarios detallados con secciones para cada uno de los 10 servicios esenciales. Cada servicio es definido en detalle, con indicadores que representan los estándares locales y estatales, y con mediciones y submediciones para cada uno de los indicadores incluidos. Después de un período de diseño de tres años, los instrumentos están siendo probados en las distintas realidades estatales y locales de los EUA.

\section{Avances en la definición de las FESP para la Región de las Américas}

En una primera etapa, el proyecto se dedicó a la tarea de examinar los avances desarrollados previamente en la definición de las FESP, para buscar coincidencias entre los diversos enfoques (11). El resultado se presenta en la figura 1.

Como se aprecia en la intersección de estos tres enfoques, hay un grado importante de coincidencia entre los avances hechos por el NPHPSP, el estudio de la OMS y la propuesta de la OPS.

Hay dos funciones en el estudio de la OMS y en la reflexión inicial de la OPS que representan campos de acción importantes de la salud pública. Usando un enfoque funcional en lugar de uno de campos de acción, estas dos funciones (salud ambiental y salud ocupacional) podrían ser tratadas como áreas de trabajo de la salud pública donde todas las FESP incluidas en la intersección de estos tres enfoques deben ser aplicadas para mejorar el funcionamiento de la salud pública en dichos campos de acción. La función de preparación para el enfrentamiento de desastres requiere acciones muy específicas que no son necesariamente parte del resto de las FESP y puede ser incluida como una función separada. 
FIGURA 1. Coincidencia entre las funciones esenciales de la salud pública en el mecanismo conceptual propuesto por la OPS, el Programa Nacional de Desempeño y Estándares de la Salud Pública (NPHPSP) y el estudio Delphi de la OMS

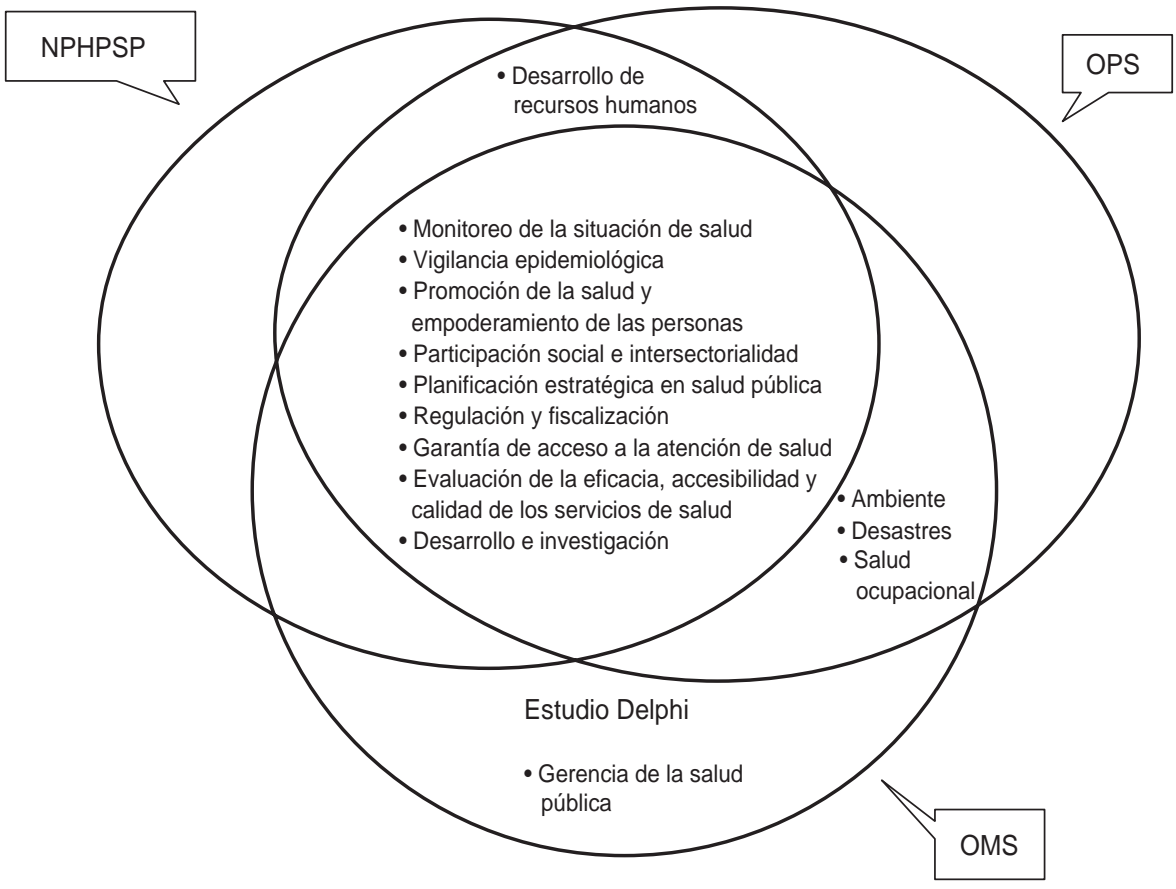

El estudio de la OMS definió una función específica para la gerencia de la salud pública que será importante incorporar para la medición en la Región de las Américas. Algo similar ocurre con el desarrollo de recursos humanos en el campo de la salud pública, incluida en el NPHPSP.

\section{LAS FESP QUE HAN SIDO IDENTIFICADAS A FIN DE MEDIR SU DESEMPEÑO EN LOS PAÍSES DE LAS AMÉRICAS}

A continuación se describen cada una de las 11 FESP que han sido identificadas como críticas para la práctica de la salud pública en los países de las Américas y que están contenidas en el instrumento de medición de su desempeño que ha sido desarrollado por la OPS en colaboración con el CDC y el CLAISS. El listado que sigue al título de cada una de ellas corresponde a los componentes que la integran y que forman la base para la definición de estándares, indicadores, mediciones y submediciones actualmente en desarrollo.

\section{Función esencial No. 1: monitoreo y análisis de la situación de salud de la población}

- Evaluación actualizada de la situación y tendencias de salud del país y de sus determinantes, con especial énfasis en la identificación de desigualdades en los riesgos, en los daños y en el acceso a los servicios.

- Identificación de las necesidades de salud de la población, incluyendo la evaluación de los riesgos de salud y la demanda de servicios de salud.

- Manejo de las estadísticas vitales y la situación específica de grupos de especial interés o mayor riesgo.

- Generación de información útil para la evaluación del desempeño de los servicios de salud.

- Identificación de recursos extrasectoriales que contribuyen a la promoción de la salud y al mejoramiento de la calidad de vida.
- Desarrollo de tecnología, experiencia y métodos para el manejo, interpretación y comunicación de la información a los responsables de la salud pública (incluyendo actores extrasectoriales, proveedores y ciudadanos).

- Definición y desarrollo de instancias de evaluación de la calidad de los datos recolectados y de su correcto análisis.

Función esencial No. 2: vigilancia de salud pública, investigación $y$ control de riesgos y daños en salud pública

- Capacidad para hacer investigación y vigilancia de brotes epidémicos y patrones de presentación de enfermedades transmisibles y no transmisibles, accidentes y exposición a sustancias tóxicas o agentesambientales nocivos para la salud.

- Infraestructura de salud pública diseñada para conducir la realización de tamizajes poblacionales, detección de casos e investigación epidemiológica en general.

- Laboratorios de salud pública capaces de conducir tamizajes rápidos y de procesar el gran volumen de exámenes necesarios para la identificación y el control de amenazas emergentes para la salud.

- Desarrollo de programas activos de vigilancia epidemiológica y de control de enfermedades infecciosas.

- Capacidad de conectarse con redes internacionales que permitan un mejor enfrentamiento de los problemas de salud de interés.

- Preparación de las autoridades nacionales de salud para activar una respuesta rápida, dirigida al control de problemas de salud o riesgos específicos.

\section{Función esencial No. 3: promoción de la salud}

- Acciones de promoción de la salud en la comunidad y desarrollo de programas para reducir riesgos $y$ daños a la salud que cuenten con una activa participación ciudadana. 
- Fortalecimiento de la intersectorialidad con el fin de hacer más eficaces las acciones de promoción de la salud, especialmente las dirigidas al ámbito de la educación formal de jóvenes y niños.

- Empoderamiento de los ciudadanos para cambiar sus propios estilos de vida y ser parte activa del proceso dirigido a cambiar los hábitos de la comunidad y a exigir a las autoridades responsables la mejoría de las condiciones del entorno para facilitar el desarrollo de una "cultura de la salud".

- Implementación de acciones dirigidas a que la ciudadanía conozca sus derechos en salud.

- Colaboración activa del personal de los servicios de salud en el desarrollo de programas educativos en escuelas, iglesias, lugares de trabajo y cualquier otro espacio de organización social relevante para proporcionar información sobre salud.

Función esencial No. 4: participación social y empoderamiento de los ciudadanos en salud

- Facilitación de la participación de la comunidad organizada en programas de prevención, diagnóstico, tratamiento y rehabilitación.

- Fortalecimiento de la construcción de alianzas intersectoriales con la sociedad civil que permitan utilizar todo el capital humano y los recursos materiales disponibles para mejorar el estado de salud de la población y promover ambientes que favorezcan la vida saludable.

- Apoyo en tecnología y experiencia a la constitución de redes y alianzas con la sociedad organizada para la promoción de la salud.

- Identificación de los recursos comunitarios que colaboren en las acciones de promoción y en la mejoría de la calidad de vida, reforzando su poder y capacidad de influir en las decisiones que afecten a su salud y a su acceso a servicios adecuados de salud pública.

- Información y defensa ante las autoridades gubernamentales en materia de prioridades de salud, en particular con relación a aquellas cuyo enfrentamiento depende de mejorías de otros aspectos del nivel de vida.

Función esencial No. 5: desarrollo de políticas, planes y capacidad de gestión que apoyen los esfuerzos en salud pública y contribuyan a la rectoría sanitaria nacional

- Desarrollo de decisiones políticas en salud pública a través de un proceso participativo en todos los niveles que resulte coherente con el contexto político y económico en que estas se desarrollan.

- Planificación estratégica a escala nacional y apoyo a la planificación en los niveles subnacionales.

- Definición y perfeccionamiento de los objetivos de la salud pública, que deben ser mensurables, como parte de las estrategias de mejoramiento continuo de la calidad.

- Evaluación del sistema de atención de salud para la definición de una política nacional que garantice la entrega de servicios de salud con enfoque poblacional.

- Desarrollo de códigos, regulaciones y leyes que guíen la práctica de la salud pública.

- Definición de los objetivos nacionales de salud pública para apoyar el papel de rectoría del Ministerio de Salud, o su equivalente, en lo que respecta a la definición de los objetivos y prioridades para el conjunto del sistema de salud.

- Gestión de la salud pública, en cuanto proceso de construcción, implantación y evaluación de iniciativas organizadas diseñadas para enfrentar problemas de salud poblacionales.

- Desarrollo de competencias para la toma de decisiones basadas en pruebas científicas y que incorporen la gestión de recursos, la capacidad de liderazgo y una comunicación eficaz.

- Desempeño de calidad del sistema de salud pública, en cuanto a resultados de la gestión exitosa, demostrable a proveedores y usuarios de los servicios.
Función esencial No. 6: regulación y fiscalización en salud pública

- Desarrollo y fiscalización del cumplimiento de los códigos sanitarios y/o las normas dirigidas al control de los riesgos de salud derivados de la calidad del ambiente; acreditación y control de la calidad de los servicios médicos; certificación de calidad de nuevos fármacos y sustancias biológicas para uso médico, equipos, otras tecnologías y cualquier otra actividad que implique el cumplimiento de leyes y reglamentos dirigidos a proteger la salud pública.

- Generación de nuevas leyes y regulaciones dirigidas a mejorar la salud, así como a promover ambientes saludables.

- Protección de los consumidores en sus relaciones con los servicios de salud.

- Ejecución de todas estas actividades de regulación de forma oportuna, $\mathrm{CO}^{-}$ rrecta, coherente y completa.

\section{Función esencial No. 7: evaluación y promoción del acceso equitativo de la población a los servicios de salud necesarios}

- Promoción de la equidad en el acceso a la atención de salud, que incluye la evaluación y la promoción del acceso efectivo de todos los ciudadanos a los servicios de salud necesarios.

- Evaluación y promoción del acceso a los servicios de salud necesarios a través de proveedores públicos o privados, adoptando un enfoque multisectorial que permita trabajar con diversos organismos e instituciones para resolver las inequidades en la utilización de los servicios.

- Desarrollo de acciones dirigidas a superar barreras de acceso a las intervenciones de salud pública.

- Facilitación de la vinculación de grupos vulnerables a los servicios de salud (sin incluir el financiamiento de esta atención) y a los servicios de educación para la salud, promoción de la salud y prevención de enfermedades. 
- Estrecha colaboración con agencias gubernamentales y no gubernamentales para promover el acceso equitativo a los servicios de salud necesarios.

\section{Función esencial No. 8: desarrollo de recursos humanos y capacitación en salud pública}

- Educación, capacitación y evaluación del personal de salud pública para identificar las necesidades de servicios de salud pública y atención de salud, enfrentar con eficiencia los problemas de salud pública prioritarios y evaluar adecuadamente las acciones de salud pública.

- Definición de requerimientos para la licenciatura de profesionales de salud en general y adopción de programas de mejoramiento continuo de la calidad de los servicios de salud pública.

- Formación de alianzas activas con programas de perfeccionamiento profesional que aseguren la adquisición de experiencias en salud pública relevantes para todos los estudiantes, educación continua en gestión y desarrollo de liderazgo en el ámbito de la salud pública.

- Desarrollo de capacidades para el trabajo interdisciplinario en salud pública.

Función esencial No. 9: garantía de calidad de los servicios de salud individual y colectivos

- Promoción de la existencia de sistemas permanentes de garantía de calidad y desarrollo de un sistema de monitoreo permanente de los resultados de las evaluaciones hechas mediante esos sistemas.

- Facilitación de la publicación de normas sobre las características básicas que deben tener los sistemas de garantía de calidad y supervisión del cumplimiento de esta obligación por parte de los proveedores de servicios.
- Existencia de un sistema de evaluación de tecnologías sanitarias que colabore en los procesos de toma de decisiones de todo el sistema de salud.

- Utilización de la metodología científica para evaluar intervenciones de salud de diferente grado de complejidad.

- Uso de este sistema para elevar la calidad de la provisión directa de servicios de salud.

Función esencial No. 10:

investigación, desarrollo e implementación de soluciones innovadoras en salud pública

- Innovación constante, que va desde los esfuerzos de investigación aplicada para impulsar cambios en las prácticas de salud pública hasta los esfuerzos de investigación científica formal.

- Desarrollo de investigación propia de las autoridades sanitarias en sus diferentes niveles.

- Establecimiento de alianzas con los centros de investigación e instituciones académicas para realizar oportunamente estudios que apoyen la toma de decisiones de las autoridades sanitarias nacionales en todos sus niveles y en lo más amplio de su campo de acción.

Función esencial No. 11: reducción del impacto de emergencias $y$ desastres en salud

- Planificación y ejecución de acciones de prevención, mitigación, preparación, respuesta y rehabilitación temprana relacionadas con la salud pública.

- Enfoque múltiple de los daños y la etiología de todas y cada una de las emergencias o desastres posibles en la realidad del país.

- Participación de todo el sistema de salud y la más amplia colaboración intersectorial en la reducción del impacto de emergencias y desastres en la salud.

\section{LA MEDICIÓN DEL DESEMPEÑO DE LAS FESP}

La idea de la medición del desempeño es la de identificar globalmente las fortalezas y las debilidades de la práctica de la salud pública, además de permitir un diagnóstico operativo de las áreas que requieren mayor apoyo para fortalecer la infraestructura de la salud pública, entendida en su más amplia acepción, esto es, incluyendo las capacidades humanas y las instalaciones y equipamientos que resultan necesarios para su buen desempeño.

Para avanzar en el logro de este objetivo resulta importante que la decisión de medir sea seguida por el desarrollo de instrumentos que puedan perfeccionarse continuamente hasta alcanzar el "óptimo razonable" que permita su utilización rutinaria en los distintos niveles en los que se ejerce la salud pública en la Región ${ }^{10}$. El desarrollo de instrumentos de medición de las FESP implica un largo proceso de definición de la función a medir, de los indicadores y estándares de desempeño o cumplimiento de la misma $\mathrm{y}$ de las mediciones y submediciones que actúan como verificadores.

\section{Definición de estándares "óptimos razonables", en lugar de "mínimos aceptables", de funcionamiento}

Como en otros procesos de medición del desempeño, debe decidirse entre definir estándares aceptables o usar estándares óptimos. La definición de niveles aceptables implica una difícil opción y es necesariamente arbitraria, tanto si se elige un nivel comparable a la hipotética realidad promedio de la Región como si se opta por lo que algún experto o grupo de expertos juzguen como mínimo necesario para el desarrollo de una función. Obviamente, la

\footnotetext{
10 Organización Panamericana de la Salud/Organización Mundial de la Salud. La salud pública en las Américas. Instrumento para la medición de las funciones esenciales de salud pública. HSP (documento mimeografiado), Washington, D.C., Abril 2000.
} 
decisión de optar por estándares óptimos debe relativizarse de acuerdo con la realidad global de la Región. Dada la heterogeneidad de este panorama, los estándares serán óptimos para las mejores condiciones que es posible demandar, en un plazo mediato, a la totalidad de los países de la Región, lo cual implica confiar en un juicio "experto" para tal efecto. Pese a ello, optar por estos óptimos razonables parece más adecuado y coherente con el objetivo de reforzar la infraestructura de la salud pública a la mayor brevedad posible.

\section{Desarrollo inicial de los instrumentos de medición}

El equipo a cargo del proyecto ha distribuido a diversos grupos de profesionales y expertos en salud pública un primer borrador del instrumento que incluye la definición de las funciones a medir. Este proceso tuvo su culminación formal en la reunión de la red de instituciones y expertos convocada por la OPS para este fin. ${ }^{11}$

Para minimizar sesgos, la validación y posterior aplicación del instrumento se harán ayudándose de la experiencia de un conjunto de personas encargadas de la toma de decisiones, procedentes de vertientes diversas de la práctica de la salud pública y de distintos niveles del sistema de salud en diversos países.

Un listado de FESP como el presentado está obviamente sujeto a errores y no puede pretender satisfacer todas y cada una de las visiones que el mundo de la salud pública tiene acerca del tema. Decisiones tales como la inclusión del empoderamiento de los ciudadanos o del estímulo de la intersectorialidad en las funciones de promoción o participación social implican algún grado de arbitrariedad, por lo que resulta imposible evitar la repetición de áreas propias de más de una función, con énfasis distintos. Obviamente, la reali-

\footnotetext{
11 Reunión de Consulta de Expertos. Funciones esenciales de salud pública y medición del desempeño de la práctica de la salud pública. Washington, D.C., 9 a 10 de septiembre, 1999.
}

dad de la práctica cotidiana de la salud pública no permite una separación absoluta de funciones, ni siquiera en el trabajo de una misma persona.

La medición de las FESP no es solo un ejercicio metodológico interesante, sino que debería permitir mejorar las prácticas de la salud pública y establecer estándares de buen funcionamiento y puntos de referencia para el mejoramiento continuo. El proceso apunta también hacia una mayor transparencia de las prácticas y servicios de salud pública y a dar mayor claridad a la generación de conocimientos y prácticas de salud pública basados en pruebas científicas. Por último, la medición debería crear la base para una mejor y mayor asignación de recursos para las acciones de salud pública.

\section{PRUEBAS PILOTO PARA LA VALIDACIÓN DEL INSTRUMENTO Y PLAN PARA SU APLICACIÓN EN LA REGIÓN}

Durante los meses de abril y mayo de 1999 se llevó a cabo un ejercicio de validación del instrumento de medición del desempeño de las FESP en tres países: Bolivia, Colombia y Jamaica. Esto se efectuó con un grupo de informantes clave que incluye funcionarios de los distintos niveles de autoridad sanitaria (central, intermedia y local), expertos académicos y representantes de sociedades profesionales de salud pública.

El instrumento está siendo ajustado en función de los resultados obtenidos en las pruebas piloto y se contará con una versión afinada a principios de septiembre de 2000. Con posterioridad se contempla su diseminación en todos los países miembros, a fin de definir mecanismos de aplicación del instrumento entre octubre de 2000 y abril de 2001.

El objetivo fundamental es que cada país lo utilice como un instrumento de auto-valoración del estado de la práctica de la salud pública, como una herramienta de diagnóstico que sea comparable entre todos los países y como un vehículo para incentivar el desarrollo de planes nacionales de mejoramiento de la práctica de la salud pública.

\section{EL USO DE LOS RESULTADOS DE LA MEDICIÓN DEL DESEMPEÑO PARA GENERAR UN PLAN DE ACCIÓN PARA MEJORAR LA PRÁCTICA DE LA SALUD PÚBLICA EN LAS AMÉRICAS}

La definición y medición de las FESP se concibe como una contribución al desarrollo institucional de la práctica de la salud pública y al mejoramiento del diálogo entre la salud pública y otras disciplinas involucradas en el quehacer de la salud. Por otra parte, una mejor definición de lo esencial debe contribuir a mejorar la calidad de los servicios y a obtener definiciones más precisas de las responsabilidades institucionales. En este sentido, parece lógico esperar que la disposición de la salud pública a rendir cuentas ante la ciudadanía de los resultados de su quehacer se inicie por lo que le es más propio y exclusivo, y no por las responsabilidades que comparte con los enfoques o disciplinas que intervienen en las decisiones generales en materia de política de salud o acerca del destino de los sistemas de salud. La legitimidad y la capacidad de convocatoria de la salud pública para el desarrollo de acciones intersectoriales debería verse fortalecida por una medición más precisa de la esencia de su quehacer.

Una mejor medición de las FESP debería permitir también una mejor cuantificación de los recursos necesarios para asegurar una infraestructura de salud pública adecuada, información que resulta esencial para gobiernos, personas responsables de tomar decisiones y agencias de cooperación internacional.

Finalmente, la definición y medición de las FESP son fundamentales para contribuir al fortalecimiento de la educación en salud pública en la Región, actividad que hoy vive una crisis en gran medida relacionada con la falta de definición de los papeles mencionados. 


\section{REFERENCIAS}

1. Lopez-Acuña D. Monitoring and evaluating health sector reform in Latin America and the Caribbean. London: Health Care International, The Economist Intelligence Unit (in press).

2. Mullan F. Don Quixote, Machiavelli and Robin Hood: public health practice, past and present. Am J Public Health 2000;90;702-706.

3. Organización Panamericana de la Salud/ Organización Mundial de la Salud. Desafíos de la educación en salud pública. Washington, D.C.: OPS/OMS; 2000.

4. Musgrove P. Public and private roles in health. World Bank Discussion Paper N ${ }^{\circ} 339$. Washington, D.C.: World Bank; 1996.

5. Organización Panamericana de la Salud/Organización Mundial de la Salud. XL Reunión Consejo Directivo. La Rectoría de los Ministerios de Salud en los procesos de reforma sectorial. Washington, D.C.: OPS/OMS; 1997.
6. Organización Panamericana de la Salud/ Organización Mundial de la Salud. II Conferencia Panamericana de Educación en Salud Pública, México, D.F., 11-13 de Noviembre de 1998. Informe. Washington, D.C.: OPS/ OMS; 1999. (Serie 15. Desarrollo de recursos humanos).

7. Bettcher DW, Sapirie S, Goon EH. Essential public health functions: results of the international Delphi study. World Health Stat Q 1998; 51:44-54.

8. Mays GP, Halverson PK, Miller CA. Assessing the performance of local public health systems: a survey of a state health agency efforts. J Public Health Manag Pract 1998;4:63-78.

9. Harrel J, Baker E. The essential services of public health. Leadersh Public Health 1994;3.

10. American Public Health Association, Association of Schools of Public Health, Association of State and Territorial Health Officials, ECS, Na- tional Association of County and City Health Officers, National Association of State Alcohol and Drug Abuse Directors, National Association of State Mental Health Program Directors, Public Health Foundation, United States Public Health Service, Agency for Healthcare Research and Quality, Centers for Disease Control, Food and Drug Administration, Health Resources and Services Administration, Indian Health Service, National Institutes of Health, Office of the Assistant Secretary for Health, Substance Abuse and Mental Health Services Administration: Report of the Public Health Functions Steering Committee, 1995.

11. Organización Panamericana de la Salud/ Organización Mundial de la Salud. 126a. Sesión del Comité Ejecutivo: funciones esenciales de salud pública. Washington, D.C., 26 al 30 de Junio de 2000. Washington, D.C.: OPS/ OMS; 2000. [CE126/17(esp)].

ABSTRACT In the Americas, health sector reforms are facing the challenge of strengthening the steering and leadership role of health authorities. An important part of that role consists of fulfilling the essential public health functions (EPHFs) that are incumbent on

\section{The essential functions of public health: an emerging theme in health sector reforms} all levels of government. For that, it's crucial to improve public health practice, as well as the instruments used to assess the current state of public health practice and the areas where it needs to be strengthened. For that purpose, the Pan American Health Organization has started an initiative called "Public Health in the Americas," with the objective of defining and measuring EPHFs, as a way of improving public health practice and strengthening the leadership provided by health authorities at all levels of government. This article summarizes conceptual and methodological aspects of defining and measuring EPHFs. The article also analyzes the implications that measuring performance on these public health roles, responsibilities, and activities would have for improving public health practice in the Americas. 